\title{
Multiple Solutions for Kirchhoff Equations under the Partially Sublinear Case
}

\author{
Wenjun Feng ${ }^{1}$ and Xiaojing Feng ${ }^{2}$ \\ ${ }^{1}$ College of Applied Mathematics, Shanxi University of Finance and Economics, Taiyuan 030006, China \\ ${ }^{2}$ School of Mathematical Sciences, Shanxi University, Taiyuan 030006, China \\ Correspondence should be addressed to Xiaojing Feng; fxj467@mail.nwpu.edu.cn
}

Received 16 June 2015; Revised 12 August 2015; Accepted 26 August 2015

Academic Editor: Gennaro Infante

Copyright (C) 2015 W. Feng and X. Feng. This is an open access article distributed under the Creative Commons Attribution License, which permits unrestricted use, distribution, and reproduction in any medium, provided the original work is properly cited.

We prove the infinitely many solutions to a class of sublinear Kirchhoff type equations by using an extension of Clark's theorem established by Zhaoli Liu and Zhi-Qiang Wang.

\section{Introduction and Main Results}

In this paper we study the existence and multiplicity of solutions for the following Kirchhoff type equations:

$$
\begin{aligned}
(a & \left.+\int_{\mathbb{R}^{N}}|\nabla u|^{2}+b \int_{\mathbb{R}^{N}} u^{2}\right)[-\Delta u+b u] \\
& =K(x) f(x, u), \quad \text { in } \mathbb{R}^{3},
\end{aligned}
$$

where $a, b$ are positive constants.

When $\Omega$ is a smooth bounded domain in $\mathbb{R}^{3}$, the problem

$$
\begin{aligned}
-\left(a+b \int_{\Omega}|\nabla u|^{2} d x\right) \Delta u & =f(x, u), \quad \text { in } \Omega \\
u & =0, \quad \text { on } \partial \Omega,
\end{aligned}
$$

has been studied in several papers. Perera and Zhang [1] considered the case where $f(x, \cdot)$ is asymptotically linear at 0 and asymptotically 4 -linear at infinity. They obtained a nontrivial solution of the problems by using the Yang index and critical group. Then, in [1] they considered the cases where $f(x, \cdot)$ is 4-sublinear, 4-superlinear, and asymptotically 4 -linear at infinity. By various assumptions on $f(x, \cdot)$ near 0 , they obtained multiple and sign changing solutions. Cheng and $\mathrm{Wu}[2]$ and $\mathrm{Ma}$ and Rivera [3] studied the existence of positive solutions of (2) and He and Zou [4] obtained the existence of infinitely many positive solutions of (2), respectively; Mao and Luan [5] obtained the existence of signed and sign-changing solutions for problem (2) with asymptotically 4-linear bounded nonlinearity via variational methods and invariant sets of descent flow; Sun and Tang [6] studied the existence and multiplicity results of nontrivial solutions for problem (2) with the weaker monotony and 4superlinear nonlinearity. For (2), Sun and Liu [7] considered the cases where the nonlinearity is superlinear near zero but asymptotically 4-linear at infinity, and the nonlinearity is asymptotically linear near zero but 4 -superlinear at infinity. By computing the relevant critical groups, they obtained nontrivial solutions via Morse theory.

Comparing with (1) and (2), $\mathbb{R}^{3}$ is in place of the bounded domain $\Omega \subset \mathbb{R}^{3}$. This makes the study of problem (1) more difficult and interesting. Wu [8] considered a class of Schrödinger Kirchhoff type problem in $\mathbb{R}^{N}$ and a sequence of high energy solutions are obtained by using a symmetric Mountain Pass Theorem. In [9], Alves and Figueiredo study a periodic Kirchhoff equation in $\mathbb{R}^{N}$; they get the nontrivial solution when the nonlinearity is in subcritical case and critical case. Liu and $\mathrm{He}[10]$ obtained multiplicity of high energy solutions for superlinear Kirchhoff equations in $\mathbb{R}^{3}$. Li et al. in [11] proved the existence of a positive solution to a Kirchhoff type problem on $\mathbb{R}^{N}$ by using variational methods and cutoff functional technique. 
In [12], Jin and Wu consider the following problem:

$$
\begin{gathered}
-\left(a+b \int_{\mathbb{R}^{N}}|\nabla u|^{2} d x\right) \Delta u+u=f(x, u), \quad \text { in } \mathbb{R}^{N}, \\
u \in H^{1}\left(\mathbb{R}^{N}\right),
\end{gathered}
$$

where constants $a>0, b>0, N=2$ or 3 , and $f \in C\left(R^{N} \times\right.$ $R, R)$. By using the Fountain Theorem, they obtained the following theorem.

Theorem A (see [12]). Assume that the following conditions hold.

If the following assumptions are satisfied,

$\left(H_{1}\right) f(x, u)=o(|u|)$ as $|u| \rightarrow 0$ uniformly for any $x \in \mathbb{R}^{N}$,

$\left(H_{2}\right)$ there are constants $1<p<2^{*}-1$ and $c>0$ such that

$$
|f(x, u)| \leq c\left(1+|u|^{p}\right), \quad \forall(x, u) \in \mathbb{R}^{N} \times \mathbb{R},
$$

where

$$
2^{*}-1= \begin{cases}\frac{N+2}{N-2}, & N \geq 3 \\ +\infty, & N=1,2\end{cases}
$$

$\left(H_{3}\right)$ there exists $\mu>4$ such that

$$
\begin{array}{r}
\mu F(x, u)=\mu \int_{0}^{u} f(x, s) d s \leq u f(x, u), \\
\quad \forall(x, u) \in \mathbb{R}^{N} \times \mathbb{R},
\end{array}
$$

$\left(H_{4}\right)$

$$
\inf _{x \in \mathbb{R}^{N},|u|=1} F(x, u)>0,
$$

$\left(H_{5}\right) f(g x, u)=f(x, u)$ for each $g \in O(N)$ and for each $(x$, $u) \in \mathbb{R}^{N} \times \mathbb{R}$, where $O(N)$ is the group of orthogonal transformations on $\mathbb{R}^{N}$,

$$
\left(H_{6}\right) f(x,-u)=-f(x, u) \text { for any }(x, u) \in \mathbb{R}^{N} \times \mathbb{R},
$$

then problem (3) has a sequence $\left\{u_{k}\right\}$ of radial solutions.

Recently, Liu and Wang [13] obtained an extension of Clark's theorem as follows.

Theorem B (see [13]). Let $X$ be a Banach space, $\Phi \in C^{1}(X$, $\mathbb{R})$. Assume $\Phi$ is even and satisfies the $(P S)$ condition, bounded from below, and $\Phi(0)=0$. If, for any $k \in \mathbb{N}$, there exists a $k$-dimensional subspace $X^{k}$ of $X$ and $\rho_{k}>0$ such that $\sup _{X^{k} \cap S_{\rho_{k}}} \Phi<0$, where $S_{\rho}=\{u \in X \mid\|u\|=\rho\}$, then at least one of the following conclusions holds.

(i) There exists a sequence of critical points $\left\{u_{k}\right\}$ satisfying $\Phi\left(u_{k}\right)<0$ for all $k$ and $\left\|u_{k}\right\| \rightarrow 0$ as $k \rightarrow \infty$.

(ii) There exists $r>0$ such that for any $0<a<r$ there exists a critical point $u$ such that $\|u\|=a$ and $\Phi(u)=0$.
Theorem A obtained the existence of infinitely many solutions under the case that $f(t, u)$ is sublinear at infinity in $u$. It is worth noticing that there are few papers concerning the sublinear case up to now. Motivated by the above fact, in this paper our aim is to study the existence of infinitely many solutions for $(1)$ when $f(t, u)$ satisfies sublinear condition in $u$ at infinity. Our tool is extension of Clark's theorem established in [13]. Now, we state our main result.

Theorem 1. Assume that $f$ satisfies $\left(H_{6}\right)$ and the following conditions:

$\left(f_{1}\right)$ There exist $\delta>0,1 \leq \gamma<2, C>0$ such that $f \in$ $C\left(\mathbb{R}^{3} \times[-\delta, \delta], \mathbb{R}\right)$ and $|f(x, z)| \leq C|z|^{\gamma-1}$.

$\left(f_{2}\right)$ Consider $\lim _{z \rightarrow 0} F(x, z) /|z|^{2}=+\infty$ uniformly in some ball $B_{r}\left(x_{0}\right) \subset \mathbb{R}^{3}$, where $F(x, z)=\int_{0}^{z} f(x, s) d s$.

$\left(f_{3}\right) K: \mathbb{R}^{3} \rightarrow \mathbb{R}^{+}$is a positive continuous function such that $K \in L^{2 /(2-\gamma)}\left(\mathbb{R}^{3}\right) \cap L^{\infty}\left(\mathbb{R}^{3}\right)$.

Then (1) possesses infinitely many solutions $\left\{u_{k}\right\}$ such that $\left\|u_{k}\right\|_{L^{\infty}} \rightarrow 0$ as $k \rightarrow \infty$.

Remark 2. Throughout the paper we denote by $C>0$ various positive constants which may vary from line to line and are not essential to the problem.

The paper is organized as follows: in Section 2, some preliminary results are presented. Section 3 is devoted to the proof of Theorem 1 .

\section{Preliminary}

In this section, we will give some notations that will be used throughout this paper.

Let $H^{1}=H^{1}\left(\mathbb{R}^{3}\right)$ be the completion of $C_{0}^{\infty}\left(\mathbb{R}^{3}\right)$ with respect to the inner product and norm

$$
(u, v)=\int_{\mathbb{R}^{3}}[\nabla u \nabla v+b u v] d x, \quad\|u\|=(u, u)^{1 / 2}
$$

Moreover, we denote the completion of $C_{0}^{\infty}\left(\mathbb{R}^{3}\right)$ with respect to the norm

$$
\|u\|_{D^{1}}^{2}=\int_{\mathbb{R}^{3}}|\nabla u|^{2} d x
$$

by $D^{1}=D^{1}\left(\mathbb{R}^{3}\right)$. To avoid lack of compactness, we need to consider the set of radial functions as follows:

$$
H=H_{r}^{1}\left(\mathbb{R}^{3}\right)=\left\{u \in H^{1}\left(\mathbb{R}^{3}\right) \mid u(x)=u(|x|)\right\} .
$$

Here we note that the continuous embedding $H \hookrightarrow L^{q}\left(\mathbb{R}^{3}\right)$ is compact for any $q \in(2,6)$.

Define a functional by

$$
J_{1}(u)=\frac{a}{2}\|u\|^{2}+\frac{1}{4}\|u\|^{4}-\int_{\mathbb{R}^{3}} K(x) F(x, u),
$$


Then we have from $\left(f_{1}\right)$ that $J_{1}$ is well defined on $H$ and is of $C^{1}$, and

$$
\begin{aligned}
\left(J_{1}(u), v\right)= & a(u, v)+\|u\|^{2}(u, v) \\
& -\int_{\mathbb{R}^{3}} K(x) f(x, u) v, \quad u, v \in H .
\end{aligned}
$$

It is standard to verify that the weak solutions of (1) correspond to the critical points of functional $J_{1}$.

\section{Proofs of the Main Result}

Proof of Theorem 1. Choose $\widehat{f} \in C\left(\mathbb{R}^{N} \times \mathbb{R}, \mathbb{R}\right)$ such that $\widehat{f}$ is odd in $u \in \mathbb{R}, \hat{f}(x, u)=f(x, u)$ for $x \in \mathbb{R}^{N}$ and $|u|<\delta / 2$, and $\widehat{f}(x, u)=0$ for $x \in \mathbb{R}^{N}$ and $|u|>\delta$. In order to obtain solutions of (1) we consider

$$
\begin{aligned}
(a & \left.+\int_{\mathbb{R}^{N}}|\nabla u|^{2}+b \int_{\mathbb{R}^{N}} u^{2}\right)[-\Delta u+b u] \\
& =K(x) \widehat{f}(x, u), \quad \text { in } \mathbb{R}^{N} .
\end{aligned}
$$

Moreover, (13) is variational and its solutions are the critical points of the functional defined in $H$ by

$$
J(u)=\frac{1}{2} a\|u\|^{2}+\frac{1}{4}\|u\|^{4}-\int_{\mathbb{R}^{3}} K(x) \widehat{F}(x, u) d x .
$$

From $\left(f_{1}\right)$, it is easy to check that $J$ is well defined on $H$ and $J \in C^{1}(H, \mathbb{R})$, and

$$
\begin{aligned}
J^{\prime}(u) v= & a(u, v)+\|u\|^{2}(u, v) \\
& -\int_{\mathbb{R}^{3}} K(x) \widehat{f}(x, u) v d x, \quad v \in H .
\end{aligned}
$$

Note that $J$ is even, and $J(0)=0$. For $u \in H$,

$$
\begin{gathered}
\int_{\mathbb{R}^{3}} K(x)|\widehat{F}(x, u)| d x \leq C \int_{\mathbb{R}^{3}} K(x)|u|^{\gamma} d x \\
\leq C\|K\|_{L^{2 /(2-\gamma)}\left(\mathbb{R}^{3}\right)}\|u\|_{L^{2}\left(\mathbb{R}^{3}\right)}^{\gamma} \leq C\|u\|^{\gamma} .
\end{gathered}
$$

Hence, it follows from (14) that

$$
J(u) \geq \frac{1}{2}\|u\|^{2}-C\|u\|^{\gamma}, \quad u \in H .
$$

We now use the same ideas to prove the (PS) condition. Let $\left\{u_{n}\right\}$ be a sequence in $H$ so that $J\left(u_{n}\right)$ is bounded and $J^{\prime}\left(u_{n}\right) \rightarrow$ 0 . We will prove that $\left\{u_{n}\right\}$ contains a convergent subsequence. By (17), we claim that $\left\{u_{n}\right\}$ is bounded. Assume without loss of generality that $\left\{u_{n}\right\}$ converges to $u$ weakly in $H$. Observe that

$$
\begin{aligned}
& \left\langle J^{\prime}\left(u_{n}\right)-J^{\prime}(u), u_{n}-u\right\rangle \\
& =a\left\|u_{n}-u\right\|^{2}+\left\|u_{n}\right\|^{2}\left\|u_{n}-u\right\|^{2} \\
& \quad+\left(\left\|u_{n}\right\|^{2}-\|u\|^{2}\right)\left(u, u_{n}-u\right) \\
& \quad-\int_{\mathbb{R}^{3}} K(x)\left(\widehat{f}\left(x, u_{n}\right)-\widehat{f}(x, u)\right)\left(u_{n}-u\right) d x .
\end{aligned}
$$

Hence, we have

$$
\begin{aligned}
& a\left\|u_{n}-u\right\|^{2} \\
& \leq\left\langle J^{\prime}\left(u_{n}\right)-J^{\prime}(u), u_{n}-u\right\rangle \\
&-\left(\left\|u_{n}\right\|^{2}-\|u\|^{2}\right)\left(u, u_{n}-u\right) \\
&+\int_{\mathbb{R}^{3}} K(x)\left(\widehat{f}\left(x, u_{n}\right)-\widehat{f}(x, u)\right)\left(u_{n}-u\right) d x \\
& \equiv I_{1}+I_{2}+I_{3} .
\end{aligned}
$$

It is clear that $I_{1} \rightarrow 0$ and $I_{2} \rightarrow 0$ as $n \rightarrow \infty$. In the following, we will estimate $I_{3}$, by using $\left(f_{3}\right)$, for any $R>0$,

$$
\begin{aligned}
& \int_{\mathbb{R}^{3}} K(x)\left|\hat{f}\left(x, u_{n}\right)-\hat{f}(x, u)\right|\left|u_{n}-u\right| d x \\
& \quad \leq C \int_{\mathbb{R}^{3} \backslash B_{R}(0)} K(x)\left(\left|u_{n}\right|^{\gamma}+|u|^{\gamma}\right) d x \\
& \quad+C \int_{B_{R}(0)} K(x)\left(\left|u_{n}\right|^{\gamma-1}+|u|^{\gamma-1}\right)\left|u_{n}-u\right| d x \\
& \quad \leq C\left(\left\|u_{n}\right\|_{L^{2}\left(\mathbb{R}^{3} \backslash B_{R}(0)\right)}^{\gamma}+\|u\|_{L^{2}\left(\mathbb{R}^{3} \backslash B_{R}(0)\right)}^{\gamma}\right) \\
& \cdot\|K\|_{L^{2 /(2-\gamma)\left(\mathbb{R}^{3} \backslash B_{R}(0)\right)}+C\|K\|_{L^{2 /(2-\gamma)}\left(B_{R}(0)\right)}}+\left(\left\|u_{n}\right\|_{L^{2}\left(B_{R}(0)\right)}^{\gamma-1}+\|u\|_{L^{2}\left(B_{R}(0)\right)}^{\gamma-1}\right)\left\|u_{n}-u\right\|_{L^{2}\left(B_{R}(0)\right)} \\
& \leq C\|K\|_{L^{2 /(2-\gamma)}\left(\mathbb{R}^{3} \backslash B_{R}(0)\right)}+C\left\|u_{n}-u\right\|_{L^{2}\left(B_{R}(0)\right)},
\end{aligned}
$$

which implies

$$
\lim _{n \rightarrow+\infty} \int_{\mathbb{R}^{3}} K(x)\left|\widehat{f}\left(x, u_{n}\right)-\widehat{f}(x, u)\right|\left|u_{n}-u\right| d x=0 .
$$

Therefore, $\left\{u_{n}\right\}$ converges strongly in $H$ and the (PS) condition holds for $J$. By $\left(f_{2}\right)$ and $\left(f_{3}\right)$, for any $L>0$, there exists $\delta=\delta(L)>0$ such that if $u \in C_{0}^{\infty}\left(B_{r}\left(x_{0}\right)\right)$ and $|u|_{\infty}<\delta$ then $K(x) \widehat{F}(x, u(x)) \geq L|u(x)|^{2}$, and it follows from (14) that

$$
J(u) \leq \frac{a}{2}\|u\|^{2}+\frac{1}{4}\|u\|^{4}-L\|u\|_{L^{2}\left(\mathbb{R}^{3}\right)}^{2} .
$$

This implies, for any $k \in \mathbb{N}$, if $X^{k}$ is a $k$-dimensional subspace of $C_{0}^{\infty}\left(B_{r}\left(x_{0}\right)\right)$ and $\rho_{k}$ is sufficiently small then $\sup _{X^{k} \cap S_{\rho_{k}}} J(u)<0$, where $S_{\rho}=\left\{u \in \mathbb{R}^{3} \mid\|u\|=\rho\right\}$. Now we apply Theorem B to obtain infinitely many solutions $\left\{u_{k}\right\}$ for (13) such that

$$
\left\|u_{k}\right\| \longrightarrow 0, \quad k \longrightarrow \infty
$$

Finally we show that $\left\|u_{k}\right\|_{L^{\infty}} \rightarrow 0$ as $k \rightarrow \infty$. Let $u$ be a solution of (13) and $\alpha>0$. Let $M>0$ and set $u^{M}(x)=$ $\max \{-M, \min \{u(x), M\}\}$. Multiplying both sides of (13) with $\left|u^{M}\right|^{\alpha} u^{M}$ implies

$$
\left.\left.\frac{4 a}{(\alpha+2)^{2}} \int_{\mathbb{R}^{3}}|\nabla| u^{M}\right|^{\alpha / 2+1}\right|^{2} d x \leq C \int_{\mathbb{R}^{3}}\left|u^{M}\right|^{\alpha+1} d x .
$$


By using the iterating method in [13], we can get the following estimate:

$$
\|u\|_{L^{\infty}\left(\mathbb{R}^{3}\right)} \leq C_{1}\|u\|_{L^{6}\left(\mathbb{R}^{3}\right)}^{v},
$$

where $v$ is a number in $(0,1)$ and $C_{1}>0$ is independent of $u$ and $\alpha$. By (23) and Sobolev Imbedding Theorem [14], we derive that $\left\|u_{k}\right\|_{L^{\infty}\left(\mathbb{R}^{3}\right)} \rightarrow 0$ as $k \rightarrow \infty$. Therefore, $u_{k}$ are the solutions of (1) as $k$ is sufficiently large. The proof is completed.

\section{Conflict of Interests}

The authors declare no conflict of interests regarding the publication of this paper.

\section{Acknowledgments}

The authors would like to express their sincere gratitude to one anonymous referee for his/her constructive comments for improving the quality of this paper. This work was supported by the National Natural Science Foundation of China (Grant nos. 11071149, 11271299, and 11301313), Natural Science Foundation of Shanxi Province (2012011004-2, 20130210014, and 2014021009-1), and the Scientific and Technological Innovation Programs of Higher Education Institutions in Shanxi (no. 2015101).

\section{References}

[1] K. Perera and Z. Zhang, "Nontrivial solutions of Kirchhoff-type problems via the Yang index," Journal of Differential Equations, vol. 221, no. 1, pp. 246-255, 2006.

[2] B. Cheng and X. Wu, "Existence results of positive solutions of Kirchhoff type problems," Nonlinear Analysis: Theory, Methods \& Applications, vol. 71, no. 10, pp. 4883-4892, 2009.

[3] T. F. Ma and J. E. M. Rivera, "Positive solutions for a nonlinear nonlocal elliptic transmission problem," Applied Mathematics Letters, vol. 16, no. 2, pp. 243-248, 2003.

[4] X. He and W. Zou, "Infinitely many positive solutions for Kirchhoff-type problems," Nonlinear Analysis: Theory, Methods \& Applications, vol. 70, no. 3, pp. 1407-1414, 2009.

[5] A. Mao and S. Luan, "Sign-changing solutions of a class of nonlocal quasilinear elliptic boundary value problems," Journal of Mathematical Analysis and Applications, vol. 383, no. 1, pp. 239243, 2011.

[6] J.-J. Sun and C.-L. Tang, "Existence and multiplicity of solutions for Kirchhoff type equations," Nonlinear Analysis: Theory, Methods \& Applications, vol. 74, no. 4, pp. 1212-1222, 2011.

[7] J. Sun and S. Liu, "Nontrivial solutions of Kirchhoff type problems," Applied Mathematics Letters, vol. 25, no. 3, pp. 500-504, 2012.

[8] X. Wu, "Existence of nontrivial solutions and high energy solutions for Schrödinger-Kirchhoff-type equations in $\mathbb{R}^{N}$," Nonlinear Analysis: Real World Applications, vol. 12, no. 2, pp. 12781287, 2011.

[9] C. O. Alves and G. M. Figueiredo, "Nonlinear perturbations of a periodic Kirchhoff equation in $\mathbb{R}^{N}$," Nonlinear Analysis: Theory, Methods \& Applications, vol. 75, no. 5, pp. 2750-2759, 2012.
[10] W. Liu and X. He, "Multiplicity of high energy solutions for superlinear Kirchhoff equations," Journal of Applied Mathematics and Computing, vol. 39, no. 1-2, pp. 473-487, 2012.

[11] Y. Li, F. Li, and J. Shi, "Existence of a positive solution to Kirchhoff type problems without compactness conditions," Journal of Differential Equations, vol. 253, no. 7, pp. 2285-2294, 2012.

[12] J. Jin and X. Wu, "Infinitely many radial solutions for Kirchhofftype problems in $\mathbb{R}^{\mathrm{N}}$," Journal of Mathematical Analysis and Applications, vol. 369, no. 2, pp. 564-574, 2010.

[13] Z. Liu and Z. Wang, "On Clark's theorem and its applications to partially sublinear problems," Annales de l'Institut Henri Poincare (C) Non Linear Analysis, 2014.

[14] M. Willem, Minimax Theorems, Birkhäuser, 1996. 


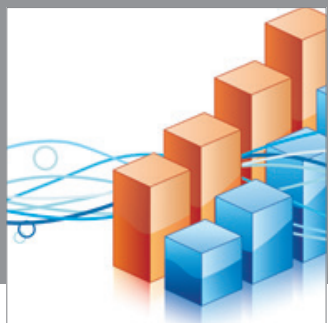

Advances in

Operations Research

mansans

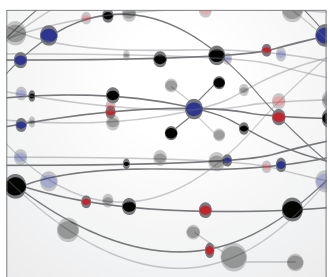

The Scientific World Journal
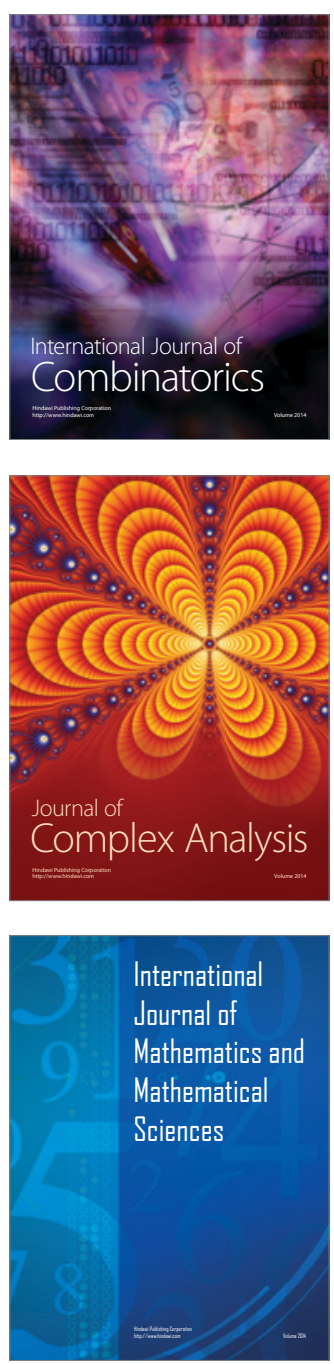
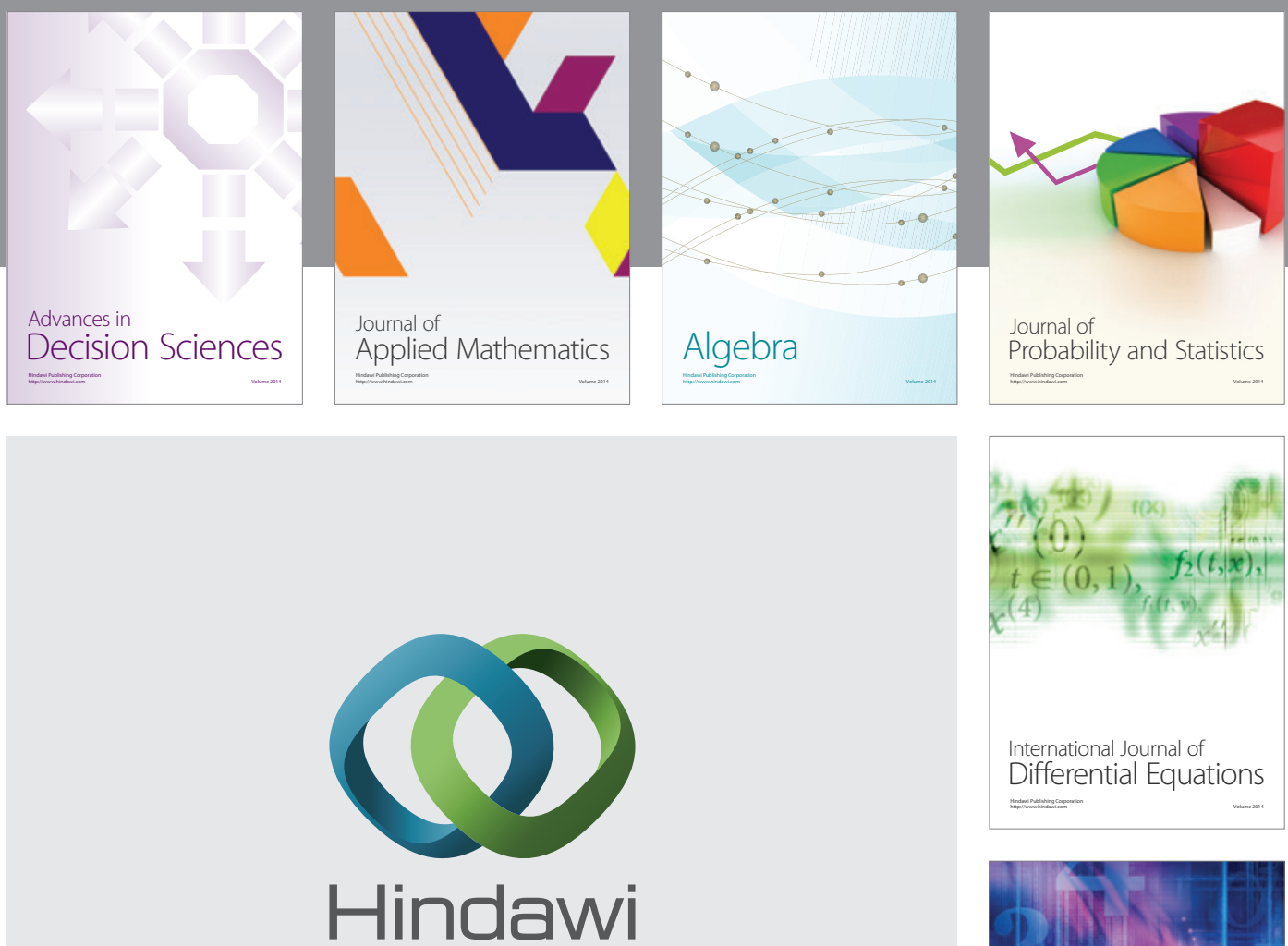

Submit your manuscripts at http://www.hindawi.com
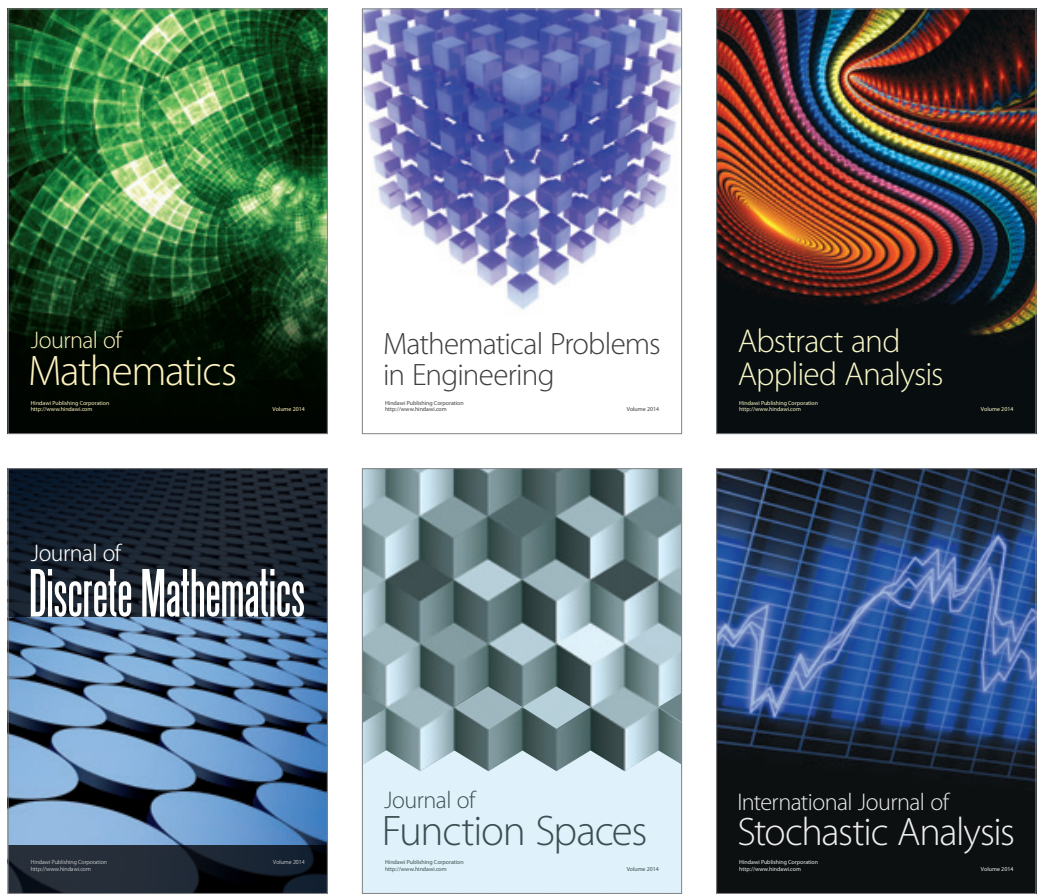

Journal of

Function Spaces

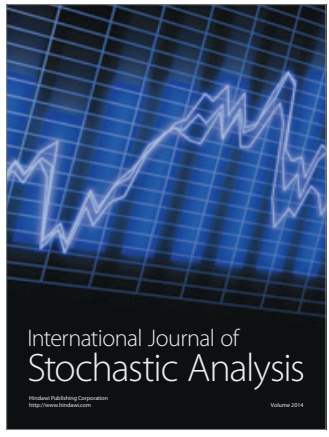

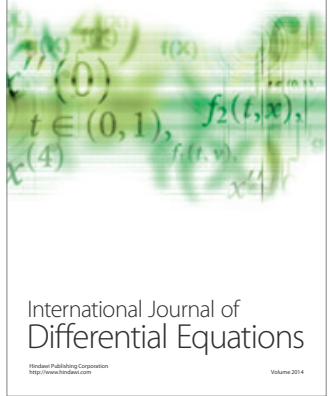
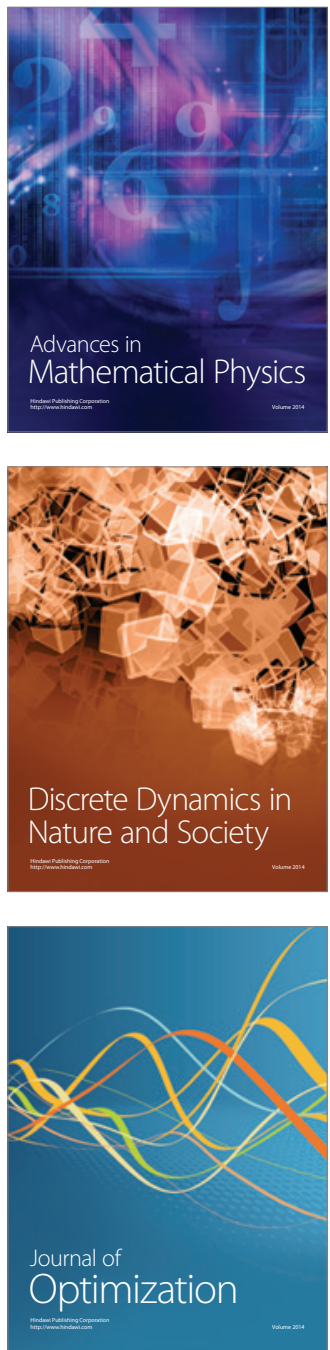